\title{
HANKEL CONVOLUTION ON THE DUAL OF A SPACE OF ENTIRE FUNCTIONS
}

\author{
JORGE J. BETANCOR
}

Abstract. In this paper we study the Hankel convolution on a certain space $\mathcal{Q}$ of entire functions rapidly increasing on each horizontal strip in the complex plane. We also describe the \#convolution operators on the dual space $\mathcal{Q}^{\prime}$ of $\mathcal{Q}$.

\section{Mathematics subject classification (2000): 46F12.}

Key words and phrases: Hankel transforms, Hankel convolution, distributions.

\section{REFERENCES}

[1] G. Altenburg, Bessel-Transformationen in Dualräumen von Grundfunktionen über dem Intervall $\Omega=(0, \infty)$ und deren Dualraumen, Math. Nachr., 108, (1982), 197-218.

[2] M. Belhadj, J. J. BetanCOR, Hankel transformation and Hankel convolution of tempered Beurling distributions, Rocky Mountain J. Math., 31, (4) (2001), 1171-1203.

[3] J. J. BetAnCor, I. MARRERo, The Hankel convolution and the Zemanian spaces $B_{\mu}$ and $B_{\mu}^{\prime}$, Math. Nachr., 160, (1993), 277-298.

[4] J. J. BETANCOR, L. RodRíGUEZ-MeSA, Hankel convolution on distribution spaces with exponential growth, Studia Math., 121, (1) (1996), 35-52.

[5] J. J. BETANCOR, L. RODRÍGUEZ-MESA, On Hankel convolutors on certain Hankel transformable function spaces, Glasgow Math. J., 39, (1997), 351-369.

[6] J. J. BETANCOR, L. RodRÍGUEZ-MESA, On Hankel convolution equations in distribution spaces, Rocky Mountain J. Math., 29, (1) (1999), 93-114.

[7] F. M. Cholewinski, A Hankel convolution complex inversion theory, Mem. Amer. Math. Soc., 58, (1965).

[8] S. J. L. VAN EIJNDHOVEn, J. DE GRAAF, Some results on Hankel invariant distribution spaces, Proc. Kon. Ned. Akad. van Wetensch., A 86, (1) (1983), 77-87.

[9] A. ERDŽLYI, Higher transcendental functions, II, McGraw Hill, New York, 1955.

[10] D. T. HAIMO, Integral equations associated with Hankel convolutions, Trans. Amer. Math. Soc., 116, (1965), 330-375.

[11] H. HASUmi, Note on the n-dimensional tempered ultradistributions, Tohoku Math. J., 13, (1961), 94-104.

[12] C. S. HeRZ, On the mean inversion of Fourier and Hankel transforms, Proc. Nat. Acad. Sci. USA, 40, (1954), 996-999.

[13] I. I. Hirschman, JR., Variation diminishing Hankel transforms, J. Analyse Math., 8, (1960/61), 307-336.

[14] I. MARRERO, J. J. BetanCOR, Hankel convolution of generalized functions, Rendiconti di Matematica, 15, (1995), 351-380.

[15] J. DE SouSA-PINTO, A generalized Hankel convolution, SIAM J. Appl. Math., 16, (1985), 1335-1346.

[16] G.N. WATsOn, A treatise on the theory of Bessel functions, Cambridge University Press, Cambridge, 1959.

[17] A. H. ZEMANIAN, A distributional Hankel transformation, J. SIAM Appl. Math., 14, (1966), 561-576.

[18] A. H. ZEMANIAN, The Hankel transformations of certain distributions of rapid growth, J. SIAM Appl. Math., 14, (4) (1966), 678-690.

[19] A.H. ZEmanian, Generalized integral transformations, Interscience Publishers, New York, 1968. 